\title{
THEORETICAL ASPECTS OF MASS LOSS FROM LATE-TYPE STARS
}

\author{
Ray J. Weymann \\ Steward observatory \\ University of Arizona \\ Tucson, Arizona 85721 U.S.A.
}

\section{INTRODUCTION}

Since Deutsch's (1956) discovery that in a Herculis the displaced circumstellar absorption lines common in the spectra of late-type giants and supergiants really represented a loss of matter, the evidence that this is a very extensive and common feature among all late giants and supergiants has become very persuasive. To the extensive optical spectroscopic evidence for mass loss from these stars originally obtained by Deutsch and subsequently by others, we may now add observations of infrared excess from dust in circumstellar envelopes (Merrill 1977), radio molecular line radiation from expanding shells (Winnburg 1971), thermal and possibly $21 \mathrm{~cm}$ radio radiation from lonized and neutral hydrogen respectively (Smolinski et al. 1977; Zuckerman 1977) and the direct photographs of the envelope of $\alpha$ Ori in the light of one of the potassium resonance lines from scattered stellar radiation (Bernat et al. 1977).

At the same time, even the answer to the fundamental theoretical question concerning the identification of the driving mechanism for the mass loss is very far from clear, and those very few detailed theoretical models for the mass flow which assume some specific mechanism are accordingly still highly suspect.

Since the purpose of this contribution is to review the state of our understanding of these mechanlsms and to comment on some of the specific models which have been proposed, and since recent comprehensive reviews of the observational aspects of this problem have been given (Goldberg 1976; Reimers 1975, 1977a) we shall summarize only those aspects of the observational material most relevant to the theoretical models, drawing heavily in what follows upon the reviews by Reimers just cited.

Nearly all theoretical models for late type stars have assumed the mass loss to occur in spherically symmetric flows. With the exception of the discussion of shockdriven mass loss in long-period variables (cf. Willson 1976; Slutz 1976), which I sha11 not discuss, the flows have also been assumed to be time-independent. It is therefore important to consider very briefly what the observational evidence for or against these two assumptions is, and whether we are being badly misled in our attempts at understanding the mass-loss mechanisms by making them. Figure $I$ is a highly schematic summary of Reimers' work, indicating in the HR diagram various regimes. To the left of the curve $A-A^{\prime}$, ground-based spectroscopic evidence for mass loss from late type giants is weak or absent, though it is not yet clear to what extent this represents a genuine drop in mass ejection rates. Since the most sensitive spectroscopic indicator of the mass loss is the $\mathrm{K}$ line of singly ionized calcium, 
this apparent decrease may be due to increasing double lonization of calcium in the circumstellar envelope. To the right of the line B-B', there seems to be very little in the way of time-dependent changes in the circumstellar envelopes. There is however some evidence of velocity fine structure in the circumstellar envelopes of these stars with a weak satellite $\sim 5$ to $10 \mathrm{~km} / \mathrm{sec}$ from the main component, but the most unambiguous case for clearly discrete multiple shells is for the very distant M supergiant $\mu$ Cep and these lines may be interstellar (Sanner 1976). In a more recent study, Sanner (1977) has shown that although there are differences in the circumstellar line strength between stars having the same effective temperature and luminosity, when very careful attempts are made to match these parameters accurately, the differences in circumstellar line strengths are not very large. These facts suggest that if time-dependent phenomena are of the essence - for example if the matter is thrown off in discrete "puffs" - then each "puff" contributes only a small portion to the mass constituting most of the column density which is observed spectroscopically. Consequently, the average time between puffs must be much less than the characteristic time for the mass in the inner regions of the envelope to be exchanged. In a star like a ori, this means that any "puffs" would need to occur at intervals of less than about a year.

of course these considerations do not exclude short term fluctuations in the flow, some of which may transport energy, but very little matter.

The same type of argument involving Sanner's study also suggests that extreme departures from spherical symetry on a large scale (large compared to the stellar radius) do not occur, for if the matter were flowing out in a thin disc one ought to see rather strong variations in the column density from star to star depending upon the aspect angle. A somewhat more convincing argument comes directly from the photographs and contour maps of the scattered potassium line radiation (Bernat et al. 1977 and references therein; Lynds et al. 1977) which in their Inner portions are quite circular. Velocity studies made in conjunction with some of these maps suggest that we are not merely looking at a disc pole on. Of course, these studies only provide Information down to a scale an order of magnitude larger than the stellar radius. (It would be fascinating, and is apparently barely feasible, to repeat such studies using speckle techniques.) Gross departures from spherical symmetry on a scale comparable to, or much smaller than, a stellar radius may well occur, as they certainly do in the sun.

In the band between $A-A^{\prime}$ and $B^{-} B^{\prime}$ the situation is different. Here the flows seem to be definitely time varfable, especially in the higher luminosities; in the lower luminosities at spectral classes late $K$ and early $M$ it is not yet clear to what extent most of the observed variability in strengths and velocity are caused by actual changes in the flow rather than by changes in the level of lonization.

Figure 1 also shows in a highly schematic way the trend of terminal velocities in the region of the HR diagram under discussion. Note in particular that in the gteady region the terminal velocities are well below $100 \mathrm{~km} / \mathrm{sec}$ and also well below 


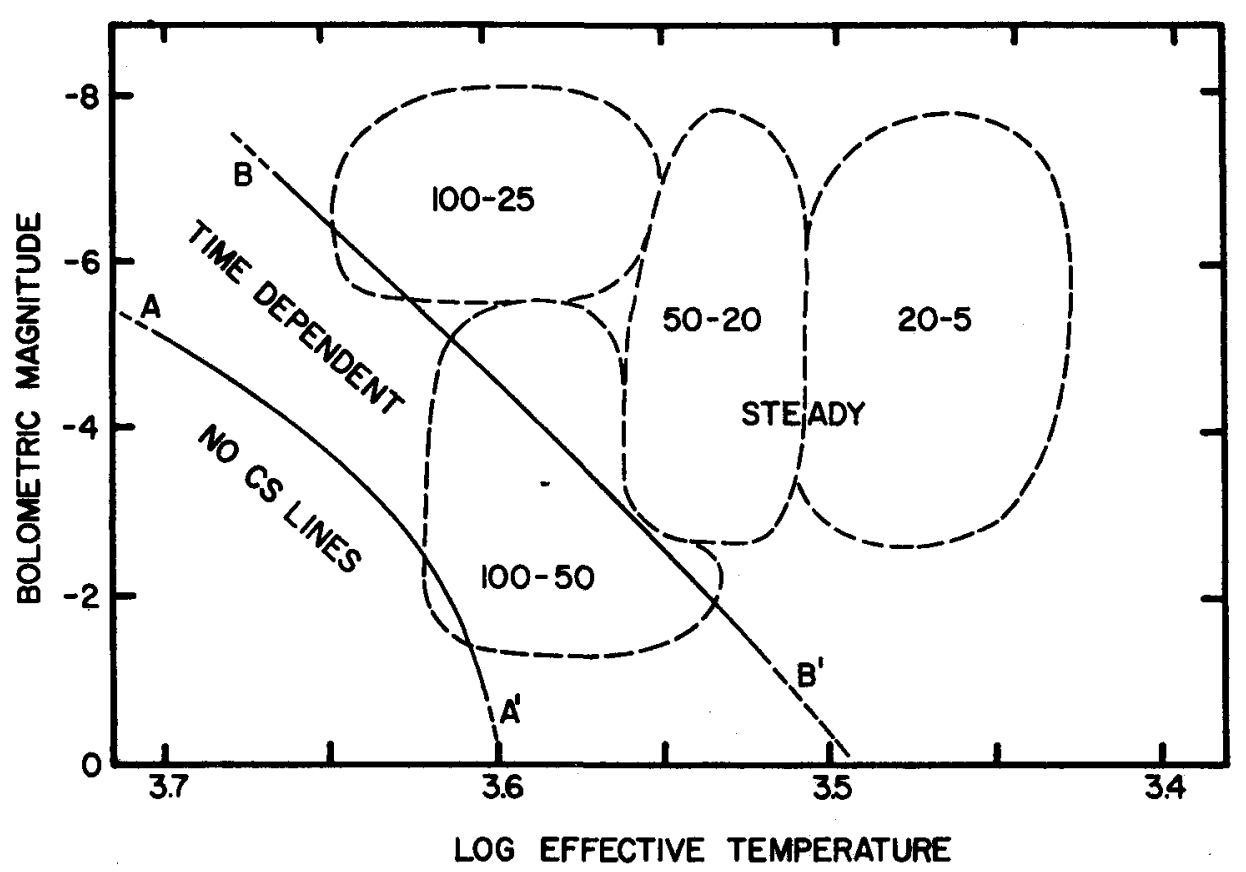

Figure 1. Mass loss domains for late-type glants in the HR diagram. (After Reimers). To the left of the line A-A' spectroscoplc evidence for mass loss (based on the $\mathrm{Ca} K$ line) is absent. In the strip between $A-A^{\prime}$ and $B-B^{\prime}$ the ejection shows evidence of time variability, while to the right of $B-B^{\prime}$ the flows seem relatively stationary. The dashed lines mark in a highly schematic way various terminal velocity regimes.

the value of the escape velocity at the stellar surfaces. (There is some suggestion from the molecular Ine studies that for very late stars the terminal velocities may Increase somewhat over those for the early and middle M stars.)

\section{DISCUSSION OF MECHANISMS}

We now consider some theoretical models for steady, spherically symmetric flows for which we have at least some hope of being applicable in the domain to the right of $B-B^{\prime}$. It is conventent to constder the varlous mechanisms for driving the mass loss according to the dominant mode of energy or momentum transport. [For a very general. theoretical discussion of stellar winds, but with special emphasis on the solar wind, see a recent review by Holzer (1977)1. There are three such modes ("thermal," "waves," "radiation") though the dominant mode may shift from one mechanism to another as one moves from layer to layer in a given star. 


\section{(a) Thermally-Driven Winds}

The success of the classic work by Parker on the solar wind driven by a hot corona (1.e. a flow in which the only momentum transport is that assoctated with thermal gas pressure) led to the hope that this single basic mechanism might also explain the spectroscopic phenomena observed in these cooler, more luminous stars. There are, however, objections to this model which have been stated before (Weymann 1962, 1963). We restate them in a slightly different way: Conslder the implications of such models for an $M$ supergiant like Orionis for which we adopt (following Gilman and Woolf 1975) $\mathrm{m} / \mathrm{m}_{\Theta}=14$ and $R / R_{\Theta}=633$, leading to an escape velocity of $92 \mathrm{~km} / \mathrm{sec}$. The terminal velocity is $10 \mathrm{~km} / \mathrm{sec}$ and the mass $10 \mathrm{ss}$ rate about $10^{-6} \mathrm{~m} / \mathrm{yr}$, though this latter figure is still quite uncertain. [Compare Reimers (1975), Sanner (1976), Bernat (1977)]. Following Parker, suppose we consider a transonic solution which is essentlally isothermal, at least through the critical point of the flow. The temperature required to produce this mass loss rate depends upon the details of the temperature rise to this "coronal" value. To take an optimistlc view, suppose an abrupt jump in temperature occurs at constant pressure starting just beyond a point where $\rho_{0}=3.5 \times 10^{-12}, \mathrm{~T}=2000$, corresponding to $\tau_{\text {cont }} z .001$ according to an atmospheric model by Johnson (1974). (To have the jump to high temperatures occur at such high densities is not realistic because, for one thing, it leaves no room for an extended warm chromosphere which is surely present as evidenced by the calcium and magnestum emission lines (see also Lambert and Snel1 1975). We then find a temperature of $27,000^{\circ} \mathrm{K}$ is required to produce the observed mass loss rate. This implies a flow of fully ionized hydrogen which will radiate far more intensely than observed. A transition to a much higher temperature at lower densities could avofd the hydrogen line emission problem, but the gas is very $\operatorname{cool}\left(\sim 1000^{\circ} \mathrm{K}\right)$ in the region where the circumstellar lines are produced. It is then hard to avoid terminal velocities much higher than observed. But even if that were possible, one would then have excessive amounts of thermal free-free radiation in $X$-rays or the UV, since all the thermal energy must be radiated away.

We concede that these remarks certainly do not constitute an alrtight argument, but we challenge those who believe that a thermally driven wind constitutes a viable mass-loss mechanism in a star like $\alpha$ Or1 to do the following: propose any run of energy deposition with radius whose accompanying flow (i) satisfies the equations of motion and continuity, (ii) produces the required mass loss rate, (111) ends up as a cool, neutral gas flowing at about $10 \mathrm{~km} / \mathrm{sec}$ at $5-20$ stellar radil from the star, (iv) does not produce an unobservably large amount of line emission or continuum emission in the radio, IR, visible, or UV. [See especially Bernat and Lambert (1976) in this latter regard].

\section{(b) Wave-Driven Winds}

If there is a fluctuating component in the velocity field, this will lead not 
only to transport of energy, but also to stresses which must be taken into account in the equation of motion. An important start in incorporating these stresses into the stellar wind formalism has recently been made by Jacques (1977) who considered Alfven and magnetosonic, as well as purely acoustic, modes. Unfortunately, it will be very hard to extend this work to the supergiants where such stresses are likely to be of most importance. In the first place, we are totally ignorant of even the magnetic field strengths in these stars (let alone their geometries), and therefore cannot even assess which mode is likely to be dominant. Secondly, the amplitudes of the fluctuations, as judged by both photospheric and chromospheric line widths, are not small, and large-amplitude fluctuations would have to be incorporated into the formalism. Finally, these line widths may have nothing whatsoever to do with progressive waves, but may be connected with large scale convective elements (Schwarzschild 1975) or non-radial osctllations (Lucy 1976a).

\section{(c) Radiative $1 y$-Driven Winds}

In contrast to the case of $O$ and $B$ supergiants, where numerous strong resonance lines of abundant elements in the far UV may drive high-velocity winds, there are too few strong atomic resonance lines in the red and near IR to drive ejection from cool stars. Some authors (Fix and Alexander 1974, Maciel 1976, 1977) have suggested that molecular opacity may be adequate, but in order to confirm this, models with the same level of detail as for the early type stars, as in, for example, Castor, Abbot and Klein (1975) need to be computed. In particular, it would be worthwile to consider only $c 0$ in considerable detall which under most circumstances is the dominant contributor (Maciel 1976).

Attention must be paid to the depletion of the level populations in the envelope as the flow cools, the density drops, and the radiation field is diluted, since the momentum transport is dependent upon the nutnber of Iines.

With the discovery of dust emission in the infrared spectra of some cool stars, attention has focused on radiative acceleration through grain opacity. This mechanism, including the question of grain-gas coupling, has been investigated by a number of authors (Lucy 1976b, Salpeter 1974, Goldreich and Scoville 1976), and in considerable detail by kwok (1975). Since the grains - especially silicates - can only form in the photospheres of the very coolest stars, this mechanism can only be of interest in explaining mass loss in the $K$ and early $M$ stars if grain formation well above the stellar surface can occur. Such a possibility was considered in a paper by Gilman and Woolf (1975).

Unfortunately this paper was never published, and we shall therefore summarize its key points: (1) It is assumed that smal1, pure magnesium silicate particles can fom as soon as the internal grain temperature drops below the vaporization temperature of about $1000^{\circ} \mathrm{K}$. (2) Under the conditions of very low density prevailing in the extended envelopes of these stars, the internal grain temperature is determined 
almost entirely by the radiation field and only very little by the local kinetic temperature (see also Jennings 1973). (3) The optical properties adopted by these authors for the grains are such that they are poor emitters and absorbers in the near IR where the stellar flux maximum occurs, but emit efficiently in the $10 \mu$ region. The grains are therefore much cooler than a blackbody at the same distance from a stellar photosphere, consequently they can survive much closer to the surface of a star than a blackbody with the same vaporization temperature. Figure 2 shows the distance from the stellar center, in units of the stellar radius, at which the GilmanWoolf grains (marked "GW") could survive as a function of the effective temperature of the stellar photospheres, assumed to radiate as blackbodies. Since the real stellar

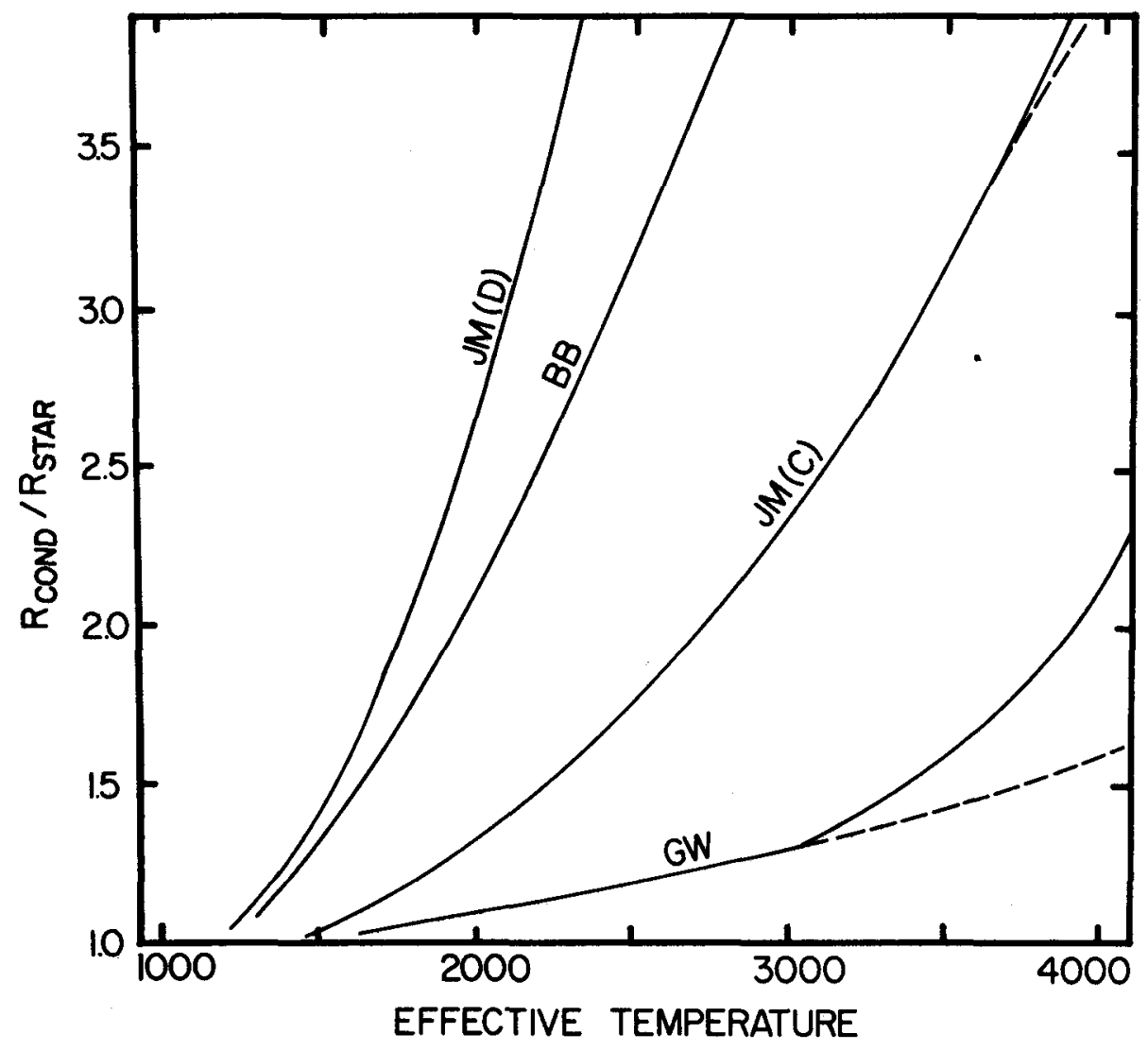

Figure 2. Condensation distance for magnesium silicate in unfts of the stellar radfus as a function of effective temperature. Condensation is assumed to occur when the equilibrium temperature of the grain is $1020^{\circ} \mathrm{K}$. Curve " $G W^{\prime}$ corresponds to the optical properties of the grains assumed by Gilman and Woolf; JM(C) and JM(D) correspond to the "clean" and "dirty" silicates discussed by Jones and Merrill, while "BB" corresponds to a grain absorbing like a black (or gray) body. The dashed lines are for the case where the stellar flux is depressed by a factor of 10 below a black body for wavelengths less than $4000 \mathrm{~A}$. 
flux distributions of late type stars have the ultraviolet severely depressed due to blanketing, the dashed extension of the GW curve shows the effect of dropping the photospheric flux by a factor of 10 below that of the blackbody curve for wavelengths below 4000\%. (4) It is assumed that grain formation takes place in the vicinity of this "survival distance" with enough efficiency that the flow is accelerated quickly through 1 ts critical point at this distance. (5) The mass loss rate is then estimated by multiplying the surface area at this distance by the sound velocity times the gas density at the critical point. The latter can be determined by integrating the momentum equation outward from the photosphere. The result of such considerations are that if only thermal pressures resulting from temperatures characteristic of the photosphere support this subsonic portion of the flow, the density scale height is In general so small that the density drops off to very low values at the "grain survival" distance and the computed mass loss rates are much smaller than the observed rates. (6) Gilman and Woolf therefore invoke the macroscopic velocity fields inferred from the chromospheric and photospheric line widths observed in these stars to provide extra momentum transport and increase the scale height. Since the data on such line widths were available in only a very few cases for which ejection rates were also available, Gilman and Woolf were able only to say that in those few cases there were no glaring qualitative discrepancies between predicted and calculated rates.

We have extended their model by "calfbrating". empirically the relation between the calcium $x$ line width (Wilson and Bappu 1957) and the "effective turbulent velocity" (hence scale height) in the outer stellar envelope by appealing to the case of the chromospheric eclipsing star $\zeta$ Aurigae (Wilson 1960; Wright 1970) where the scale height in the chromosphere can be estimated. In this way we can predict mass loss rates throughout this region of the HR diagram and compare these rates with Reimer's spectroscopically estimated empirical rates. The details of this extension will be discussed elsewhere, but this procedure does correctly predict qualitatively the trend of the mass loss rates and also generally gives the correct rates to within an order-of-magnitude or so which is about all that we can expect from such a crude procedure.

\section{(d) Criticism of the Dust-Briven Mass Loss Mechanism}

Despite the modest success of the model described above, this mechanism is subject to several crittcisms which we first enumerate and then comment upon: Statistically, the $\mathrm{H}$ and $\mathrm{K}$ emission is displaced blueward with about $40 \%$ of the circumstellar line terminal velocity. This suggests that in the chromosphere itself (where it is felt that no grains ought to exist), mass loss is already underway and therefore some acceleration mechanism other than grain acceleration must play. a dominant role. (2) In at least one very low metal abundance red giant, HD 221170 , there is a circumstellar K line (Reimers 1977b). Additionally, there is also evidence for mass loss from $\mathrm{H \alpha}$ emission in some population II giants (Cohen 1976). It 
is then argued that insufficient grain formation would occur in such low-metal abundance environments to drive the mass loss. (3) Some arguments, both direct and indirect, suggest that grain condensation doesn't occur as close to the stellar surface as assumed in the Gilman-Woolf mode1. In the case of IRC 10011, lunar occultation measurements at 2 microns led Zappala et al. (1974) to the conclusion that no dust had condensed within 3 stellar radii. Moreover, Jones and Merril1 (1976) found that it was necessary to increase the near IR absorbtivity of the grains over that for pure Mg silicates in order to convert sufficient stellar flux to produce the IR excess at $10 \mu$ observed in certain stars. This increased absorbtivity makes the grains hotter and pushes out the "survival distance."

We now briefly comment on these three difficulties in reverse order:

(3) If the actual optical properties of typical silicates shortly after formation are those given by Jones and Merrill (1976) for their case of "dirty silicates," the increase in the survival distance is so marked that the Gilman-Woolf proposal becomes untenable. This increase is shown by the curve in Figure 2 marked JM(D). Even the parameters assumed by Jones and Merr1l1 for the "clean" silicates [JM(C)] lead to condensation distances distinctly larger than the Gilman-Woolf values. For comparison we have also plotted in Figure 2 the survival distance for blackbodies. These same considerations are relevant to the interpretation of the lunar occultation data: If the grains are extremely poor emitters at $2 \mu$ and scatter mostly forward. they could survive within 3 stellar radii, but an optically thin shell would then be very hard to detect at $2 \mu$. On the other hand, sufficiently dirty grains would not be expected to exist within about 3 stellar radii even for an object as cool as IRC 10011, as Figure 2 suggests.

There are two possible escapes from this difficulty. First, it is conceivable that the impurities tend to be added after the grains have grown somewhat and cooled somewhat. Secondy, it is possible that some of the 10 micron excess ascribed to dust is really chromospheric in origin (Lambert and Snel1 1975) and the grains need not be nearly as dirty as those described by Jones and Merrill.

(2) More data are needed to clarify quantitatively the relationship between metal abundance and mass loss rate for objects of the same effective temperature and luminosity. Grains are exceedingly efficient absorbers and a quantitative investigation needs to be made of just how low the metal abundance must drop before the grains provide insufficient opacity and coupling to drive the mass loss. It should also be borne in mind that for a young population I star and an extrenely old population II star both having the same effective temperature and luminosity, the population II star will have a distinctly lower surface gravity.

(1) The fact that a measurable net outflow occurs in the chromospheric regions (usually tacitly assumed to occur below the region of grain condensation) does not necessarily conflict with the dust-driven flows described above. In fact, in the approximation in which the grains condense in a very thin layer during whtch accel- 
eration through the critical point occurs the flows must become nearly sonic - 1.e. reach a speed of a few $\mathrm{km} / \mathrm{sec}$ - before the grains condense, and it is the gradient in the "turbulent pressure" which is responsible for this. Nevertheless, in this model it is still the radiative force acting on the grains over large distances which does the bulk of the work against gravity. Additionally, if it is true that the grain temperature is determined almost entirely by radiative processes, then grains could very well survive in, and co-exist with mild chromospheric temperatures in the range $4000-8000^{\circ} \mathrm{K}$ provided the density is very low. In fact, Jennings (1973) suggests that grains not only survive, but can quench chromospheric temperatures. It is interesting in this connection to note that Sanner (1977) also suggests an anti-correlation between the intensity of $\mathrm{H}, \mathrm{K}$ emission and the circumstellar line strength.

It has been objected that while grains might be able to survive a chromospheric environment, they could not form in it. The objection can be made more general than this: Until the condensation process can be followed from the molecular level through to the grains, it isn't clear whether the radiative properties of the material at each step of the condensation process allow or prevent further growth in the non-equilibrium environment of low denstites and dilute radiation field.

It is not the intent of the foregoing remarks to defend the grain-driven mechanism "at all costs," but simply to point out that the objections are not as decisive as sometimes assumed.

(e) The Criticism by R. N. Thomas and Collaborators

R. N. Thomas and his collaborators [cf. Cannon and Thomas (1976) and references therein] have criticized both the thermally-driven and radiatively-driven mechanisms described above, although some of their criticism is directed more specifically at radiatively-driven flows in hot stars. The main assertions made are: (1) It is maintained that the origin of the flow and its character may be critically affected by the presence of a net mass flux in the subphotospheric layers through the influence of this mass flux on (for example) the generation of acoustic flux, and that this influence must be carefully investigated before the flows can be understood. (2) Radiative processes may influence the terminal velocity of the flow but cannot "Initiate" it. (3) The transonic flows passing through the critical point are in a sense artifically imposed, "over-constrain" the flow and are not the ones actually realized in nature. Instead it is suggested that "would-be" transonic flows are unstable to the generation of acoustic waves and that their dissipation prevents or seriously delays the flows from beconing supersonic.

The third criticism is perhaps the heart of the matter, since if point (3) is not valid, one can then hardly argue that criticisms (1) and (2) are valid, at least in the case of the dust-driven model. It is true that no one has ever explicitly demonstrated (by either analytic or numerical methods) that the time-dependent evolution of an originalily static atmosphere in which, for example, dust grains condense, will in fact asymptotically approach the steady trans-sonic flows. Investigation of 
this problem would be an instructive, though difficult, undertaking.

III. SOME SPECULATIONS ON CORONAS AND TRANSIENT FLOWS

The observations of the circumstellar material in the strip A-A', B-B', particularly at the higher luminosities, show much more evidence for time-dependence than in the $M$ supergiants. It seems very reasonable that stars not too distant in the HR diagram from the sun have hot coronae which will drive solar-type winds and it is of interest to ask where and why this mechanism gives way to some other mechanism - for example dust-driven mass loss - which is surely the most plausible mechanism in the extremely cool stars observed via the $\mathrm{OH}$ masers. This question is obviously connected with the question of which stars have coronae and how hot they should be, a question on which a number of authors have worked. One of the recent discussions is by Hearn (1975), whose model was applied to late-type stars by Mullan (1976). Hearn examfned the total energy loss associated with a corona with some base pressure $P_{0}$ and found that this loss was minimized for some coronal temperature $T_{c}$, the value of $T_{c}$ depending upon $P_{0}$ and the stellar mass and radius as well. Hearn's crucial argument, involving thermal stability, is that this minimum temperature $T_{c}\left(P_{o}, M, R\right)$ is the one realized in nature. $P_{o}$ (and hence $T_{c}$ ) is then determined by setting the total coronal energy loss equal to the (assumed) energy input in the form of mechanical dissipation. We would argue that, details aside, this argument is not correct because it enables one to deduce the pressure and temperature of the corona without any reference to the momentum balance (i.e. hydrostatic equilibrium in a first approximation) which surely must be invoked to connect the photospheric pressure with the coronal pressure.

It is a striking fact that to date no UV observations have turned up clear evidence for any temperatures characteristic of the solar transition zone or corona (i.e. $T \geq 10^{5}$ ) in giants of $c l$ ass $K$ or $M$, and equally striking that even the moderately hot chromospheric temperatures required to produce HeI 10830 are lacking in $M$ giants and supergiants (Linsky 1976). Evidently, the $M$ giants and supergtants are not able to surmount the thermal barrier presented by the high emissivity of hydrogen and helium in the range $2-8 \times 10^{40} \mathrm{~K}$ and the $j u m p$ to comparable emissivity at very much higher temperatures $\left(T \geqslant 10^{6} \mathrm{~K}\right)$ is never made - or if it is, not until very low densities are reached. Whether this comes about mainly because of differences in the quantity and character of the non-thermal energy deposition between the sum and the $M$ stars, or whether $1 t$ is partly simply a matter of low surface gravity maintaining moderately high gas denstities, cannot yet be said. In any event, we speculate that in the $A-A^{\prime} / B-B^{\prime}$ transition region incipient transient coronas occur. We further speculate that there are large amounts of gas which alternate back and forth between the hot and cold phases of these incipient coronae and that the cold condensations, partly confined and accelerated by a much lower density hot phase, may account for some of the spectroscopic phenomena in this transition region. The arguments made earlier against uniform thermally driven winds do not apply equally in this case 
since we may be seeing here cold condensations embedded in a spectroscopically invisible rarified hot flow, rather than the cooled remnants of the hot flow itself. However, the main motivation for this speculation is not so much to explain the transient phenomena typified by a star like $\alpha$ Tau (Reimers $1977 \mathrm{~b}$ ), but rather to stimulate work in understanding the cool condensations which are clearly present (and sometimes moving at high velocities) in the envelopes of some stars, most notably in the envelope of the chromospheric eclipsing binary 31 Cygni (Wright 1970).

\section{SUMMARY}

We have reviewed the mechanisms proposed to account for the mass loss observed to occur throughout the upper right portion of the Hertzsprung-Russell diagram. Not surprisingly, we conclude that no single mechanism is free of major objections. We speculate that the transient phenomena associated with $G$ supergiants and $K$ II giants may be associated with incipient, transient coronae and condensations induced by thermal instabilities. In the extremely luminous supergiants the very large macroscopic velocity fields inferred from both chromospheric and photospheric line profiles seem certain to play an important role. We are hindered by our woeful ignorance of the character of the motion these widths represent. Dust-driven mass loss, coupled with "turbulent support" of the inner envelope out to the dust-condensation point still seems the most promising mechanism for the M stars. This mechanism would fail however, if the nature of the grain and the grain condensation process are such that they cannot form quite close ( -1.5 stellar radii from the center) to the surface. If it should prove that such nearby condensation cannot occur, then we are in a real theoretical dilemna, for in the $M$ giants - in a star like B Peg for example - there is simply no observable mechanism able to deliver the required momentum and energy to get the matter away from the star.

In such an eventuality we should then probably have to turn to localized activity analogous to solar flares and prominences. If a study of the solar surface teaches us anything, it teaches us how severe are the departures from spherical symmetry and steady state conditions on a sma11 scale. Th1s will be extremely hard to mode1 theoretically and extremely hard to pursue observationally. The most promising approach would seem to be continued work (now supplemented by UV observations) on the chromospheric eclipsing binaries pioneered by Wright and others, where we have at least some chance of studying this small-scale structure.

I am very much indebted to the large number of astronomers who have kindly brought me up-to-date on this field by discussions and written communications. These include: Drs. Bernat, Boesgaard, Castor, Feldman, Fix, Gilman, Goldberg, Hagen, Holzer, Klein, Kwok, Merri11, Lambert, Linsky, Lucy, Lynds, Reimers, Sanner, Thomas, Woolf, and Zuckerman. 
REFERENCES

Bernat, A. P.: 1977, Astrophys. J., 213, 756.

Bernat, A. P., Honeycutt, R. K., Kephart, J. E., Gow, C. E., Sandford, M. T., and Lambert, D. L.: 1977, in preparation.

Bernat, A. P., and Lambert, D. L.: 1976, Astrophys. J., 204, 830.

Cannon, C. J. and Thomas, R. N.: 1977, Astrophys. J., 211, 910.

Castor, J. I., Abbott, D. C., and Klein, R. I.: 1975, Astrophys. J., 195, 157.

Cohen, J. G.: 1976, Astrophys. J. Letters, 203, L127.

Deutsch, A. J.: 1956, Astrophys, J., 123, 210.

Fix, J. D. and Alexander, D. R.: 1974, Astrophys. J. Letters, 188, 191.

Gllman, R. C. and Woolf, N. J.: 1975 (unpublished).

Goldberg, L.: 1976, Mem. Soc. Roy. Sc1. Liege 6th Ser. 9, 387.

Goldreich, P. and Scoville, N.: 1976, Astrophys. J., 205, 144.

Hearn, A. G.: 1975, Astron. and Astrophys., 40, 355.

Holzer, T. E.: 1977, to appear in Solar System Plasma Physlcs: A Twentieth Anniversary Overview. Edited by C. F. Kenne1, L. J. Lanzerotti and E. N. Parker, North-Holland Publishing Company.

Jacques, S. A.: 1977, Astrophys. J., 215, 942.

Jennings, M. C.: 1973, Astrophys. J., 185, 197.

Johnson, H. C.: 1974, NCAR Technical Note NCAR-TN/STR-95, Boulder, Col.

Jones, T. W. and Merrill, K. W.: 1976, Astrophys. J., 209, 509.

Kwok, S.: 1975, Astrophys. J., 198, 585.

Lambert, D. L. and Sne11, R. L.: 1975, Monthly Notices Roy. Astron. Soc., $172,277$.

Linsky, J. L.: 1976, The Study of Solar Output and Variability Viewed in the Broader Context of Stellar Activity: A Critical Review to be published in The Solar Output and its Variation, 1975.

Lucy, L.: 1976a, Astrophys. J., 206, 499.

Lucy, L.: 1976b, Astrophys. J., 205, 482.

Lynds, C. R., Harvey, J. W. and Goldberg, L.: 1977, Bull. American Astron. Soc., 9, 345 .

MacleI, J. W.: 1976, Astron. and Astrophys., 48, 27.

Macie1, J. W.: 1977, Astron. and Astrophys., 57, 273.

Merri11, K. M.: 1977, Infrared Observations of Late Type Stars in IAU Colloquium No. 42, Bamberg.

Mullan, D. J.: 1976, Astrophys. J., 209, 171.

Reimers, D. 1975: in Problems in Stellar Atmospheres and Envelopes, edited by B. Baschek, W. H. Kegel, G. Traving, Springer-Verlag, Berlin-Heldelberg-New York p. 229 .

Reimers, D.: 1977a, Optical Information on Mass-Loss From Evolved Stars in IAU Colloquium No. 42, Bamberg.

Reimers, D.: 1977b, Astron. and Astrophys., 57, 395. 
Salpeter, E. E.: 1974, Astrophys. J., 193, 585.

Sanner, F.: 1976, Astrophys. J. Supp., 32, 115.

Sanner, F.: 1977, Mass Loss, Variability, and Chromospheric Activity (preprint).

Schwarzschild, M.: 1975, Astrophys. J., 195, 137.

Slutz, S.: 1976, Astrophys. J., 210, 750 .

Smolinski, J., Feldman, P. A. and lliggs, L. A.: 1977, Bul1. American Astron. Soc., 9. 344.

Weymann, R.: 1962, Astrophys. J., 136, 476.

Weymann, R.: 1963, Ann. Rev. Astron. and Astrophys., $1,97$.

Wilson, O. C. and Bappu, M. K. V.: 1957, Astrophys. J., 125, 661.

Wilson, 0. C.: 1960, in Stellar Atmospheres, P. 436. Edited by J. L. Greenstein, Vo1. VI of Stars and Stellar Systems, edited by G. P. Kuiper and B. M. Middlehurst, University of Chicago Press.

Willson, L. A.: 1976, Astrophys. J., 205, 172.

Winnberg, A.: 1977, Molecular Observations of Late-Type Stars in IAU Colloquium No. 42, Bamberg.

Wright, K. O.: 1970, in Vistas in Astronomy, 12, 147. Edited by A. Beer, Pergamon Press.

Zappala, R. R., Becklin, E. E., Matthews, K. and Neugebauer, G.: 1974, Astrophys. J., 192,109 .

Zuckerman, B.: 1977, private communication.

\section{I S C U S S I O N Of paper by WEYMANN:}

RENZINI: You spent some time in order to show that grains can survive close to the stellar surface; actually the problem is whether they can form. It seems to me that in order to have grain formation, the gas temperatuxe must be smaller than the vapour/solid phase transition temperature, which is between 1000 and $1500 \mathrm{~K}$. Whether in red giants the temperature minimum between the photosphere and the chromosphere is below about $1000-1500 \mathrm{~K}$ is not known, but seems unlikely, except perhaps in most extreme cases (e.g., very cold carbon stars). Therefore, the most likely place for grain formation seems to ne far away from the stellar surface, once that the gas has been cooled below the phase transition temperature. If this is the case, grain formation is a consequence of mass loss rather than the contrary.

MERRILL: In response to the request to inferred substantial absorption of silicates of stellar radiation (Jones and Merrill 1976), I want to point out that this conclusion was arrived at in an attempt to match observations to radiative transfer calculations in circumstellar envelopes. "Clean" (relatively non-abscrbing) grains could not match observations. To fit the entire range of dust shells observed, required that the grains, 
where they are emitting substantial flux, must be "dirty" (relatively good absorbers of the cool stellar radiation). As noted by Weymann, this may not necessarily describe the grains where they first form. Conceivably a mantle might rapidiy accrete on an initially "clean" condensation core. However, the inferred high near-IR cross-section for the grains is clearly a strong constraint on grain formation and mass-loss theories.

R.N. THOMAS: I make three quick comments (details can be read in Thomas, Astron. Astrophys., Dec. 1973; Cannon and Thomas, Ap. J., Febr. 1977):

1. The logic of the "universal" association of mass-loss (massflux), chromospheres, and some emission lines is the following:

a. empirically, the observed mass-fluxes all across the HR diagram require velocities to reach sonic velocitles at $R / R$ (phot) $<1.04$ (empirical value).

b. theoretically, (i) if one imposes hydrostatic and radiative equilibrium, one can make a model for any ( $T$ eff, $g$ ) that matches the interstellar medium at infinity; (ii) if one inludes a flux of mechanical energy from sub-atmosphere convection, as solution one gets a chromospheric convection with, following Parker, a wind $=F_{\text {mass }} ;$ (iii) this requires going back to the convection, to introduce $F_{\text {rad, }} F_{\text {mechan, }} F_{\text {mass }}$ as parameters to be determined. These then give (a) above.

2. The boundary condition $v=v_{\text {therm }}$ (or $v_{\text {sonic }}$ ) at $v=v_{e s c}$, applied by Parker and copied in subsequent discussions, imposes that the star is a perfect wind tunnel; but for a given "nozzle shape", which is the star, this condition is not generally satisfied. In general, in the stellar case, one gets a "shock" before the "nozzle throat" reaches the critical point, which corresponds to chromospheric heating and leads to (la) above.

3. One can match Wilson-Bappu profiles and the (luminosity, width) relation by combination of an optically thick chromosphere and a systematic, not turbulent, outward accelerating velocity field. No turbulence is necessary.

The overall criticism of Weymann's otherwise objective and good sumary is that he does not ask "what are the observational characteristics required to specify the star as an open, nonthermal, nonlinearly nonEquilibrium, thermodynamic system". 